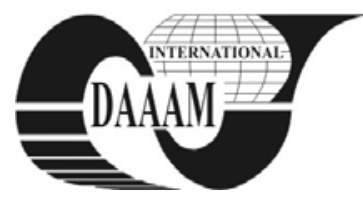

Annals of DAAAM for 2011 \& Proceedings of the 22nd International DAAAM Symposium, Volume 22, No. 1, ISSN 1726-9679 ISBN 978-3-901509-83-4, Editor B. Katalinic, Published by DAAAM International, Vienna, Austria, EU, 2011 Make Harmony between Technology and Nature, and Your Mind will Fly Free as a Bird Annals \& Proceedings of DAAAM International 2011

\title{
BEHAVIOR OF WEB SAFE COLORS WITH DIFFERENT PRINT PRODUCTION PROFILES
}

\author{
ZITINSKI ELIAS, P[aula]; POLJICAK, A[nte]; STRGAR KURECIC, M[aja] \& MANDIC, L[idija]
}

\begin{abstract}
In the area of colour reproduction there is the imperative of shifting between the monitor display colour model and the one used in reproduction. Additionally, different printing substrates have different printing capabilities, so their profiles change accordingly. The goal is to be able to obtain the same image independently of the media it is displayed in. For this purpose colour profiles are used. In this paper we observe web safe colours under three separate colour profiles in order to understand the limitations of each of them. Results show that colours shift significantly between profiles.
\end{abstract}

Key words: web safe colours, colour profiles, CIE $L^{*} a^{*} b^{*}, \Delta E$ value

\section{INTRODUCTION}

Web safe colours, also known as browser safe palette, are a set of 216 colours that are displayed exactly the same on all computer monitors. Nevertheless, when wanting to achieve the same colour reproduction on a printing substrate, a digital conversion must be made. This digital conversion is referred to as colour management, and its primary goal is to obtain a good match across colour devices by shifting between colour profiles.

In this paper the sRGB colour space was observed and compared to CMYK coated and uncoated paper colour space. The 216 web safe colours were observed in their standardized values and under the three different profiles mentioned.

Measurements in printing and colour space used in graphic technologies in the context of colour definition, as well as the tolerance between two colours, are defined by CIE L*a*b* colour space (Zjakić, 2007). The value L* represents lightness, $a^{*}$ is the red-green axis, and $b^{*}$ blue-yellow axis.

With the results of the CIE $\mathrm{L} * \mathrm{a}^{*} \mathrm{~b} *$ values, the $\Delta E$ formula was used to calculate the objective differences of the 216 safe colours between the sRGB and the coated paper profile and the sRGB and the uncoated paper profile. The $\Delta E$ results show that colour management produces differences when shifting between profiles. In addition, one can observe that the changes in colours when printing onto an uncoated printing substrate are greater than when printing onto a coated printing substrate.

Future work goes in direction of additional input profile research, pursuing the goal of finding an optimal profile which will recreate the input colours as accurately as possible.

\section{COLOUR MANAGEMENT}

As the need of a close description of colours arises, abstract colour models are used. A colour model can be explained as an abstract mathematical model describing the way colours can be represented as tuples of numbers, typically as three or four values or colour components. When this model is associated with a precise description of how the components are to be interpreted, the resulting set of colours is called colour space (Ganesan et al., 2011). The most common colour models are RGB, CMYK, YIQ and HSI.
In the RGB colour model the primary colours are red, green and blue. This model is found in digital devices such as computer monitor displays, cameras and television, where colour mixture is achieved through photons of light, and colour synthesis is an additive, as white colour is achieved through adding the three primary components.

The CMYK colour model is made out of cyan, magenta, yellow and black and is suited for printing reproduction. It is a subtractive model, i.e. by primary component subtraction, white colour is obtained. On the other hand, when mixing them, a dark shade is achieved, since adding different pigments causes various colours not to be reflected and thus not to be seen.

The YIQ model was designed to separate chrominance from luminance, a requirement in the early days of colour television. The $\mathrm{Y}$ channel represents the luminance information, while I and Q carry colour information.

In the HSI colour model $\mathrm{H}$ stands for hue, $\mathrm{S}$ for saturation and I for intensity (luminance). Hue and saturation of colours respond closely to the way humans perceive it, thus this model is well suited for an interactive manipulation of colour images where changes occur for each variable shift that corresponds to what the operator expects (Ganesan et al., 2011).

A complete subset of colours which can be represented in a given space is called gamut. The larger the gamut, the larger is the colour space.

CIE $\mathrm{L}^{*} \mathrm{a} * \mathrm{~b} *$ (CIELAB) is a generic, device independent colour space, intended for equal perceptual differences in the $\mathrm{L}^{*}, \mathrm{a}^{*}$ and $\mathrm{b}^{*}$ coordinates. It was specified by the International Commission on Illumination. The three coordinates of CIE $\mathrm{L}^{*} \mathrm{a} * \mathrm{~b}^{*}$ represent the lightness of the colour $\left(\mathrm{L}^{*}=0\right.$ is black and $\mathrm{L}^{*}=100$ indicates diffuse white; specular white may be higher), its position between red/magenta and green $\left(\mathrm{a}^{*}\right.$, where negative values indicate green while positive values indicate magenta) and its position between yellow and blue ( $b^{*}$, where negative values indicate blue and positive values indicate yellow). CIE L*a*b* is based on the concept that colours can be considered as combinations of red and yellow, red and blue, green and yellow, and green and blue (Ganesan, 2011). This colour space is shown in figure 1 .

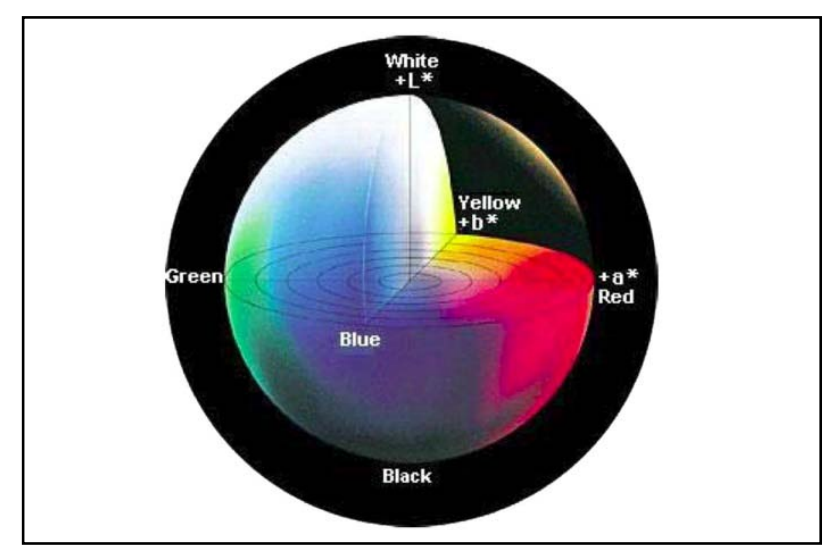

Fig. 1. CIE L*a*b* colour space 
Unlike RGB and CMYK colour models, CIE L*a*b* is designed to approximate human vision. Another characteristic of the CIE $\mathrm{L}^{*} \mathrm{a}^{*} \mathrm{~b} *$ system is that the distance that can be calculated between two colours is directly proportional to the difference between the two colours as perceived by the human eye (Ganesan, 2011).

These differences can be measured as a mathematical distance between two colours, and are referred to as $\Delta E$, as can be seen in the equation (1).

$$
\Delta E=\sqrt{\left(L_{2}-L_{1}\right)^{2}+\left(a_{2}-a_{1}\right)^{2}+\left(b_{2}-b_{1}\right)^{2}}
$$

With the aid of $\Delta E$ calculations differences between two colours can be measured, which enables to quantify the difference of one colour in two different colour profiles.

Colour profiles are numerical models of colour spaces. It is usual for operating systems and programs to have a colour profile, as it allows them to interpret the colour in their colour space (Anderson \& Krogh, 2011). Since most colour spaces are limited, i.e. their gamut is smaller than a human-based, device independent colour space, a conversion between colour spaces needs to be made in order to achieve the best suited colour replacement in a given colour space. This is why we employ the term "colour management", i.e. switching between colour profiles.

Colour management can be defined as the "communication of the associated data required for unambiguous interpretation of colour content data, and application of colour data conversions as required to produce the intended reproductions" (Green, 2010).

\section{METHODOLOGY}

A colour comparison between profiles was made for this research and web safe colours in three colour profiles were observed.

Web safe colours were chosen in the first place because of their uniformity in monitor displays. They are composed of three bytes, each for a red, green and blue channel. Safe colours consist of 216 colours that are displayed solid and consistent on any computer monitor screen capable of displaying at least 8bit colour. One can see these colours in Adobe Photoshop, as shown in figure 2.

Since the safe colour values are often displayed in hexadecimal and/or RGB colour space, so a conversion to CIE L*a*b* was made using MathWorks Matlab R2009b. This conversion was necessary, as CIE $\mathrm{L}^{*} \mathrm{a} * \mathrm{~b} *$ colour space is designed to approximate human vision (Ganesan et al., 2011).

A comparison was made when shifting from profile number 1 to profile number 2 (suited for coated substrates), and when shifting from profile number 1 to profile number 3 (suited for uncoated substrates). The colour conversion intent used was relative colourimetric rendering.

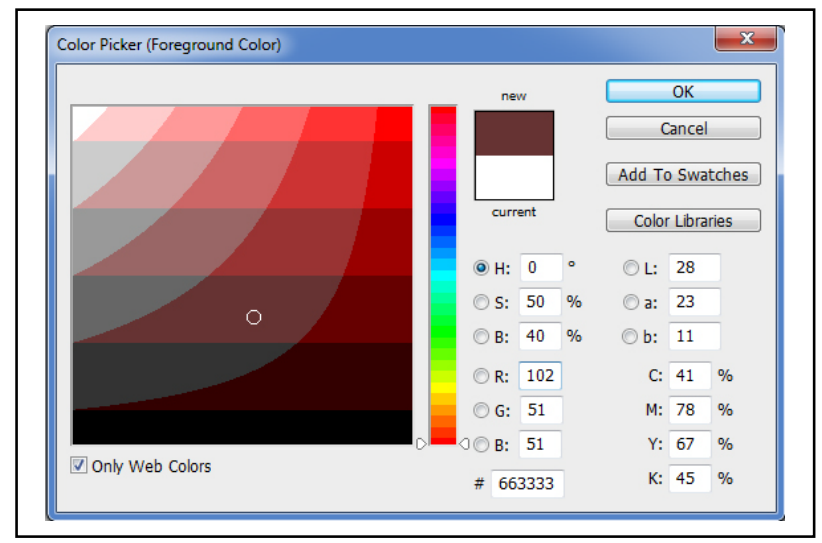

Fig. 2. Web safe colours
This rendering maintains smooth colour gradation by compressing the entire tonal range, not destroying colour information, but redistributing it.

\section{RESULTS AND DISCUSSION}

A total of 216 web safe colours were observed in the three different profiles described in section 3 . The results for the chosen representative $8 \mathrm{RGB}$ and CMYK colours + white are displayed in table 1 .

\begin{tabular}{|l|c|c|}
\hline & $\Delta E$ (sRGB - coated) & $\Delta E$ (sRGB - uncoated $)$ \\
\hline Red & 10.61 & 9.89 \\
\hline Green & 58.37 & 70.13 \\
\hline Blue & 51.64 & 54.65 \\
\hline Cyan & 42.59 & 47.07 \\
\hline Magenta & 27.43 & 28.50 \\
\hline Yellow & 4.45 & 6.17 \\
\hline Black & 0 & 0.74 \\
\hline White & 0 & 0 \\
\hline
\end{tabular}

Tab. 1. Results of the $\Delta E$ values

Results show a high level of discrepancy between profiles, shown in high $\Delta E$ values. The highest values show the green, blue and cyan colours, the red and magenta colours show medium alterations, while yellow shows low alterations. Black and white show no alteration in $\Delta E$ values in case of white or extremely low $\Delta E$ values in case of black, unnoticeable to the bare eye as it is below 1 (Zjakić, 2007).

In addition, one can notice a similarity between colour values in the profiles suited for coated and uncoated paper. Profile 3 generally shows the highest $\Delta E$ values, while $\Delta E$ values in profile 2 are usually somewhat lower.

The mean $\Delta E$ value in profile 2 was calculated in 14.23; whilst in profile 3 the value was greater (17.08).

In the case of possible printing reproduction, if the intention is to get some similar colour impression, it must be noted that the safe colours must be very carefully chosen, and the appropriate rendering intent has to be selected.

\section{CONCLUSION}

Web safe colours were observed under three different colour profiles - profile number 1 (sRGB IEC61966-2.1), profile number 2 (coated Fogra27 1S0 12647-2:2004) and profile number 3 (uncoated Fogra29 ISO 12647-2:2004).

Some safe colours, like other special or custom colours, can be precisely reproduced in all systems, for which reason they deserve special attention.

\section{REFERENCES}

Anderson, R. \& Krogh, P. (2011). Colour Space and Colour Profiles, Available from:

http://dpbestflow.org/colour/colour-space-and-colourprofiles Accessed: 2011-07-12

Ganesan, P.; Rajini, V. \& Rajkumar, R.I. (2010). Segmentation and edge detection of colour images using CIELAB colour space and edge detectors, Available from: http://0ieeexplore.ieee.org.cisne.sim.ucm.es/stamp/stamp.jsp?tp=\& arnumber $=5706186$ Accessed: $2011-08-25$

Green, P. (2010). Colour Management, Understanding and Using ICC Profiles, John Wiley \& sons, Ltd, ISBN: 978-0470-05825-1, West Sussex, United Kingdom

Zjakić, I. (2007). Upravljanje kvalitetom ofsetnog tiska, Hrvatska sveucilisna naklada, ISBN: 9531691452, Zagreb, Croatia

*** (2011) http://www.mathworks.com/ - TheMathworks Accessed on: 2011-02-11 